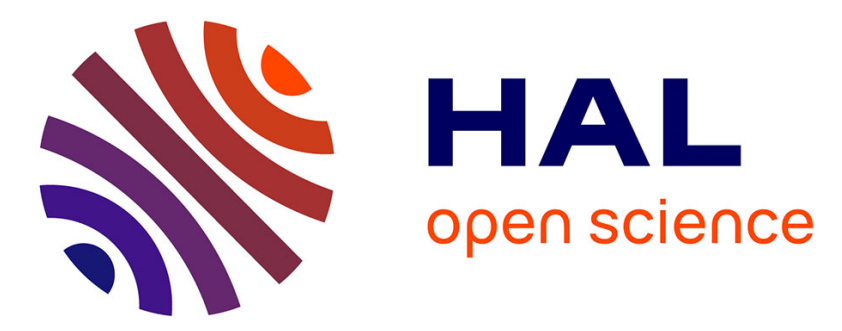

\title{
Biais de sélection dans les enquêtes postales : une étude sur le devenir des couples traités par fécondation in vitro (FIV)
}

Pénélope Troude, Estelle Bailly, Juliette Guibert, Jean Bouyer, Elise de La Rochebrochard

\section{To cite this version:}

Pénélope Troude, Estelle Bailly, Juliette Guibert, Jean Bouyer, Elise de La Rochebrochard. Biais de sélection dans les enquêtes postales: une étude sur le devenir des couples traités par fécondation in vitro (FIV). Epidemiology and Public Health = Revue d'Epidémiologie et de Santé Publique, 2012, 60 (Suppl. 2), pp.S52. 10.1016/j.respe.2012.06.028 . hal-02422870

\section{HAL Id: hal-02422870 https://hal.science/hal-02422870}

Submitted on 23 Dec 2019

HAL is a multi-disciplinary open access archive for the deposit and dissemination of scientific research documents, whether they are published or not. The documents may come from teaching and research institutions in France or abroad, or from public or private research centers.
L'archive ouverte pluridisciplinaire HAL, est destinée au dépôt et à la diffusion de documents scientifiques de niveau recherche, publiés ou non, émanant des établissements d'enseignement et de recherche français ou étrangers, des laboratoires publics ou privés. 
Troude Pénélope, Bailly Estelle, Guibert Juliette, Bouyer Jean, La Rochebrochard Elise (de), 2012, «Biais de sélection dans les enquêtes postales : une étude sur le devenir des couples traités par fécondation in vitro (FIV) », Revue d'Épidémiologie et de Santé Publique, 60(Suppl2), p. S52. DOI: $10.1016 /$ j.respe.2012.06.028.

\title{
Biais de sélection dans les enquêtes postales : une étude sur le devenir des couples traités par fécondation in vitro (FIV)
}

\author{
Pénélope Troude ${ }^{a, b, c}$, Estelle Bailly ${ }^{a}$, Juliette Guibert ${ }^{d}$, Jean Bouyer ${ }^{b}$, Elise de la Rochebrochard ${ }^{a, b}$ \\ a Institut National d'Etudes Démographiques (Ined), Paris, France \\ b Université Paris-Sud, UMRS 1018, Le Kremlin-Bicêtre, France \\ c Hôpital Lariboisière, AP-HP, Paris, France \\ d Laboratoire de procréation médicalement assistée, Institut Mutualiste Montsouris, Paris, France
}

Introduction. Dans les enquêtes, la non-participation peut avoir des conséquences importantes sur la validité des résultats. L'objectif de ce travail était d'étudier les facteurs associés au contact et à la réponse dans une enquête postale portant sur le devenir de couples ayant initié un programme de fécondation in vitro (FIV).

Méthodes. L'enquête DAIFI a inclus 6507 couples ayant débuté un programme de FIV en 2000-2002 dans l'un des huit centres de FIV participant à l'étude. Les données médicales des couples et leur parcours dans le centre ont été obtenus à partir des dossiers médicaux des centres de FIV pour les 6507 couples. L'information sur le devenir des couples après le départ du centre a été obtenue par questionnaire postal auprès des couples en 2008-2009.

Résultats. Au total, $62 \%$ des couples ont pu être contactés et $58 \%$ d'entre eux ont répondu au questionnaire postal (taux de participation : $36 \%$ ). La naissance d'un enfant au cours du traitement dans le centre était significativement associée à la probabilité de contact et à celle de réponse. Cette 
association persistait après ajustement sur les autres facteurs associés tels que l'âge de la femme, l'origine de l'infertilité et les caractéristiques à la première tentative.

Discussion et conclusion. La non-réponse mais également le non-contact pourraient être associés à la variable d'intérêt (réalisation du projet parental à long terme). Cette étude montre que les hypothèses a priori peuvent être trop simplistes et sous-estimer les biais. Des méthodes telles que l'imputation multiple doivent alors être envisagées lors de l'analyse. 